\title{
Analysis of Capture Trajectories into Periodic Orbits About Libration Points
}

\author{
Masaki Nakamiya* \\ Graduate University for Advanced Studies, Kanagawa 229-8510, Japan \\ Daniel J. Scheeres $\ddagger$ \\ University of Michigan, Ann Arbor, Michigan 48109 \\ Hiroshi Yamakawa \\ Kyoto University, Gokasho, Uji, Kyoto 611-0011, Japan \\ and \\ Makoto Yoshikawa $\$$ \\ Japan Aerospace Exploration Agency, Kanagawa 229-8510, Japan
}

DOI: $10.2514 / 1.33796$

\begin{abstract}
Spacecraft capture trajectories to Lyapunov/Halo periodic orbits of the $L 1$ and $L 2$ points in the restricted Hill three-body problem are analyzed. The specific focus is on transfer to these orbits from interplanetary trajectories. This application is motivated by future proposals to place "deep space ports" at the Earth and Mars $L 1$ or $L 2$ points. We use stable manifolds for capture trajectories to periodic orbits around the libration points. Numerical results show that the stable and unstable manifolds from periodic orbits around the libration points can intersect the surface of any of the planets of the solar system by changing the size of periodic orbits. Applying this to Earth-Mars transfers, the cost of capture into a periodic orbit is reduced compared with direct capture into a parabolic orbit. Moreover, if a spaceport is built on a periodic orbit in the vicinity of a sun-Mars libration point and propellant can be supplied there to the spacecraft, the required $\Delta V$ for entry into a circular orbit about Mars from an interplanetary trajectory can be considerably reduced compared with a direct entry into a circular orbit.
\end{abstract}

\section{Introduction}

$\mathbf{S}$ INCE the start of the space age when Sputnik was launched by the former Soviet Union in 1957, there have been many space exploration missions to the moon, Mars, Jupiter, and other solar system bodies. Future exploration plans include human missions to Mars and the continued exploration of asteroids and comets with the goals of understanding the formation process of the solar system and the origin of life. Because of these goals, there have been many investigations of interplanetary transfer trajectories. For a spacecraft to depart from Earth and escape, it is usually necessary to use a highthrust propulsion system. On the other hand, in the case of interplanetary transfers, it is often expedient to use a propulsion system with high specific impulse, like electric propulsion, to reduce the required propellant onboard a spacecraft. Therefore, to establish a reusable and repetitive interplanetary cargo system, it is mass effective to split the high-thrust and low-thrust portion of a transfer into two systems. This means that a high-thrust propulsion system is used from the ground to a relay point located at the boundary of the Earth's gravitational dominance, and then the payload is changed to another spacecraft that has a propulsion system of high specific

Presented as Paper 228 at the the AAS/AIAA Spaceflight Mechanics Meeting, Sedona, AZ, 28 January-1 February 2007; received 31 July 2007; revision received 30 December 2007; accepted for publication 5 February 2008. Copyright (C) 2008 by the American Institute of Aeronautics and Astronautics, Inc. All rights reserved. Copies of this paper may be made for personal or internal use, on condition that the copier pay the $\$ 10.00$ per-copy fee to the Copyright Clearance Center, Inc., 222 Rosewood Drive, Danvers, MA 01923; include the code 0731-5090/08 \$10.00 in correspondence with the CCC.

${ }^{*}$ Ph.D. Candidate, Department of Space and Astronautical Science; nakamiya@isas.jaxa.jp. Student Member AIAA.

${ }^{\dagger}$ Associate Professor, Department of Aerospace Engineering; currently Professor, University of Colorado, Department of Aerospace Engineering Sciences; scheeres@colorado.edu. Associate Fellow AIAA.

Professor, Research Institute for Sustainable Humanosphere; yamakawa@rish.kyoto-u.ac.jp. Senior Member AIAA.

${ }^{\S}$ Associate Professor, Institute of Space and Science; makoto@isas. jaxa.jp. Member AIAA. impulse for an interplanetary transfer. Thus, there has been great interest in the libration points as a candidate location for such a relay point for transportation.

The libration points of the circular restricted three-body problem are located where the gravity of the first and second massive bodies and centrifugal force are balanced. In particular, the position of $L 1$ and $L 2$, which lie on the line connecting the two masses, can be considered equivalent to the boundary of the gravitational dominance of the smaller mass of the two. Because an object around these points can maintain the same position with respect to the two masses, transfers between the primary body and the libration point have been investigated extensively in the past [1-6]. In fact, several astronomical satellites have already used such Halo/ Lissajous orbits around the $L 1$ and $L 2$ points of the sun-Earth system [7], and large astronomical observatories like the James Webb Space Telescope (JWST) will likely be located near the sun-Earth $L 2$ point requiring human servicing and repair [8]. Moreover, a transfer to the inner or outer planets is relatively simple by addition of energy to a spacecraft in the vicinity of the $L 1$ and $L 2$ points. Therefore, these points are also considered as candidate gateways for interplanetary transfers in the future [8-12]. Recently, the analysis and design of transfer orbits using invariant manifolds associated with periodic orbits around the libration points have been a topic of study [13-19]. Ross et al. studied the use of the Earth-moon $L 1$ point as the staging node for further human expeditions $[\underline{9}, 10]$. Farquhar and Dunham described a plan to use the sun-Earth $L 2$ libration point as the primary hub for future human space activities in the Earth's neighborhood [11,12]. The Japan Aerospace Exploration Agency (JAXA) has started investigating a deep-space port built in the vicinity of the $L 2$ point of the sun-Earth system. The escape trajectories from the libration points of sun-Earth system have also been examined [20-22].

Furthermore, if a spaceport is also built around the $L 1$ or $L 2$ points of a target celestial body, the fuel required for landing or for entry orbit at the target celestial body can be supplied there to the spacecraft arriving from interplanetary space, or we can transfer cargo there to

\footnotetext{
I JAXA Vision: JAXA 2005; http://www.jaxa.jp/2025/index_e.html.
} 


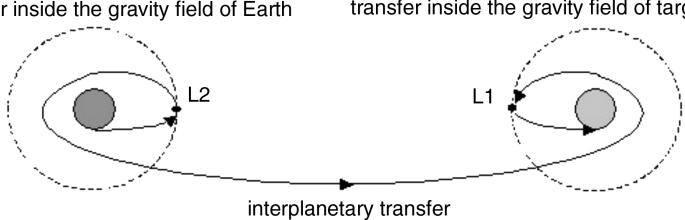

Fig. 1 Vision of an interplanetary transfer in the future.

another spacecraft which is used exclusively for landing or for an entry orbit [11]. In this way, constructing a spaceport in the vicinity of $L 1$ or $L 2$ of the sun-Earth and sun-target celestial body system can separate the transportation system into three regions: transfer inside the gravity field of the Earth, transfer inside the gravity field of a target celestial body, and the interplanetary transfer phase (see Fig. 1). Moreover, this system facilitates round-trip exploration using spaceports as relay points and leads to a reusable transportation system [8-11]. In the past, capture trajectories from/to the secondary body in the three-body problem were studied [23-28]. However, capture trajectories from an interplanetary trajectory to the vicinity of the $L 1$ and $L 2$ points of a target celestial body are not fully understood. Thus, spacecraft capture trajectories to periodic orbits of the $L 1$ and $L 2$ points in the restricted Hill three-body problem are analyzed in this paper. Although the capture to quasi-periodic orbits may result in even greater fuel savings, we will first analyze capture trajectories to a Lyapunov/Halo orbit to outline our procedure and then consider the quasi-periodic orbit transfer in the future. The specific focus is on transfer into the periodic orbits of libration points from interplanetary trajectories, although our study also sheds light on the escape problem as well.

\section{Hill Three-Body Model}

The physical system considered in this paper is the restricted Hill three-body model. This model is a limiting case of the circular restricted three-body problem (CR3BP) and describes the dynamics of a massless particle attracted by two point masses revolving around each other in a circular obit (see Fig. 2). In fact, the Hill model can be obtained from the CR3BP by setting the center of the coordinate system to be at the secondary body and then assuming that the distance of the satellite from the center is small compared to the distance between the target body and the sun. The resulting equations of motion provide a good description for the motion of a spacecraft in the vicinity of the $L 1$ and $L 2$ libration points of the smaller body [29].

\section{A. Equations of Motion}

The equations of motion for a spacecraft in the Hill model are given by $[30,31]$

$$
\begin{gathered}
\ddot{x}-2 \omega \dot{y}-3 \omega^{2} x=-\frac{\mu}{r^{3}} x \\
\ddot{y}+2 \omega \dot{x}=-\frac{\mu}{r^{3}} y \\
\ddot{z}+\omega^{2} z=-\frac{\mu}{r^{3}} z
\end{gathered}
$$

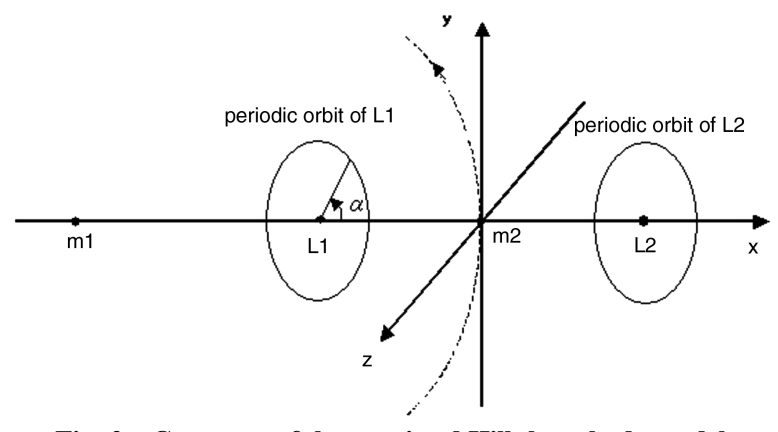

Fig. 2 Geometry of the restricted Hill three-body model. where $r=\sqrt{x^{2}+y^{2}+z^{2}}$ is the distance from the center of a second smaller body to a spacecraft, $\omega$ is the angular velocity of the secondary body $m 2$ about the primary body $m 1$, and $\mu$ is the gravitational parameter of the secondary body.

\section{B. Libration Points}

In the circular restricted three-body problem model there are five points where the gravity of the first and second massive bodies and centrifugal force acting on the spacecraft $(\mathrm{S} / \mathrm{C})$ are balanced, which are called libration points. In the restricted Hill three-body problem, only the $L 1$ and $L 2$ libration points exist, and they are symmetric to the origin with coordinates $x= \pm\left(\mu / 3 \omega^{2}\right)^{1 / 3}, y=z=0$.

\section{Jacobi Integral}

Equations (1-3) have an integral of motion similar to the CR3BP. The following equation denotes the Jacobi integral, which is a conservative quantity determined from the initial conditions

$$
J=\frac{1}{2} v^{2}-\frac{\mu}{r}+\frac{1}{2} \omega\left(3 x^{2}-z^{2}\right)
$$

where $v=\sqrt{\dot{x}^{2}+\dot{y}^{2}+\dot{z}^{2}}$ is the velocity of the particle in the rotating frame. This constant has a deep influence on the dynamics of motion. The condition $v^{2} \geq 0$ in Eq. (4) imposes a restriction on the allowable position for the motion at any given value of $J$. Setting $v=0$ defines the zero-velocity surface, which sets a physical boundary of the allowable motion at a given value of $J$. In particular, the critical value of $J$ at $L 1$ and $L 2$ defines the energy at which the zero-velocity surfaces open at $L 1$ and $L 2$, and is expressed as

$$
J_{L 1,2}=-\frac{1}{2}(9 \mu \omega)^{2 / 3}
$$

\section{Normalization}

Next, we normalize the preceding equations setting the unit length and the unit time as follows:

$$
l=\left(\frac{\mu}{\omega}\right)^{1 / 3} \quad \text { and } \quad \tau=\frac{1}{\omega}
$$

The normalized equations of motion are then

$$
\ddot{x}-2 \dot{y}-3 x=-\frac{x}{r^{3}}
$$

$$
\ddot{y}+2 \dot{x}=-\frac{y}{r^{3}}
$$

$$
\ddot{z}+z=-\frac{z}{r^{3}}
$$

This normalization allows us to eliminate all free parameters from the equations, thus, computations performed for them can be scaled to any physical system by multiplying by the unit length and time, which only depend on the properties of the primary and secondary bodies. Moreover, this normalization is equivalent to $\omega=1$ and $\mu=1$, thus, the normalized $x$ coordinate of libration points and the normalized value of $J$ at $L 1$ and $L 2$ are equal to

$$
\begin{gathered}
x= \pm(1 / 3)^{1 / 3}= \pm 0.693 \ldots \\
J_{L 1,2}=-(1 / 2) 9^{2 / 3}=-2.16337 \ldots
\end{gathered}
$$

Table 1 gives the normalized radius for the planets of the solar system. This is a quantity of interest, as it defines the closest periapsis passage possible to the planet, and defines the periapsis radius where significant drag forces are available for an aerocapture spacecraft. 
Table 1 Normalized radius of the solar system planet

\begin{tabular}{lcccc}
\hline \hline Planet & Mass, $\mathrm{kg} \times 10^{23}$ & $\begin{array}{c}\text { Gravitational parameter, } \\
\mathrm{km}^{3} / \mathrm{s}^{2} \times 10^{5}\end{array}$ & $\begin{array}{c}\text { Mean motion, } \\
\mathrm{rad} / \mathrm{s} \times 10^{-7}\end{array}$ & Normalized radius \\
\hline Mercury & 0.3302 & 0.220329 & 8.27 & 0.007663 \\
Venus & 4.869 & 3.248889 & 3.24 & 0.00415 \\
Earth & 5.9742 & 3.986345 & 1.99 & 0.002955 \\
Mars & 0.64191 & 0.428321 & 1.06 & 0.002173 \\
Jupiter & 1899 & 1267.127 & 0.168 & 0.000933 \\
Saturn & 568.8 & 379.5375 & 0.0676 & 0.000641 \\
Uranus & 86.86 & 57.9582 & 0.0267 & 0.000253 \\
Neptune & 102.4 & 68.32742 & 0.0121 & 0.000148 \\
\hline \hline
\end{tabular}

\section{E. Periodic Orbits in the Vicinity of the Libration Points}

There exist periodic orbits near the libration points in the two- and three-dimensional space [32-36] called Lyapunov and Halo orbits, respectively, whose sizes depend on the value of the Jacobi constant. If a spaceport is built on a Halo orbit about the $L 2$ point, it is not hidden in the shadow of the second body because the radius of the Halo orbit can be made larger than that of the second body. However, we will first analyze capture trajectories to a planar Lyapunov orbit to outline our procedure and then consider the Halo orbit transfer case later.

We compute the Halo orbit as follows: First, we assume the initial condition is $X_{0}=\left(x_{0}, 0, z_{0}, 0, \dot{y}_{0}, 0\right)$. Using the Runge-Kutta fourthorder method, the equations of motion are integrated keeping seventh-order accuracy of the Jacobi constant until the sign of $y$ changes twice, and the time at this point is defined to be $t$. If $X_{t}=\left(x_{0}, 0, z_{0}, 0, \dot{y}_{0}, 0\right)$, that orbit is considered to be a Halo orbit $(t$ is considered to be a period of Halo orbit $T$ at this time). If the orbit does not close on itself at $t$, we use a minimization algorithm for nonlinear functions [37] to drive the norm of the difference $X_{t}-X_{0}$ to zero.

\section{F. Invariant Manifold}

There exist invariant structures associated with these periodic orbits called unstable and stable manifolds [38,39]. These are trajectories that depart from or wind onto the periodic orbit, depending on whether they are stable or unstable, respectively. We exploit these stable manifolds for capture trajectories to periodic orbits around the libration points (see Fig. 3). In this paper, invariant manifolds are generated by applying an infinitesimal impulse (0.00001: corresponds to $1.66 E-3 \mathrm{~m} / \mathrm{s}$ in the sun-Mars system) at different locations along the periodic orbit and integrating backward in time. The location on the periodic orbit is parameterized by a phase angle $\alpha$ on the periodic orbit (see Fig. 2).

\section{Capture Trajectories to the Vicinity of $L 1$ and $L 2$}

\section{A. Assumption of Capture Trajectories}

In this paper, we assume that capture trajectories are trajectories that enter the sphere of influence of a target body from interplanetary

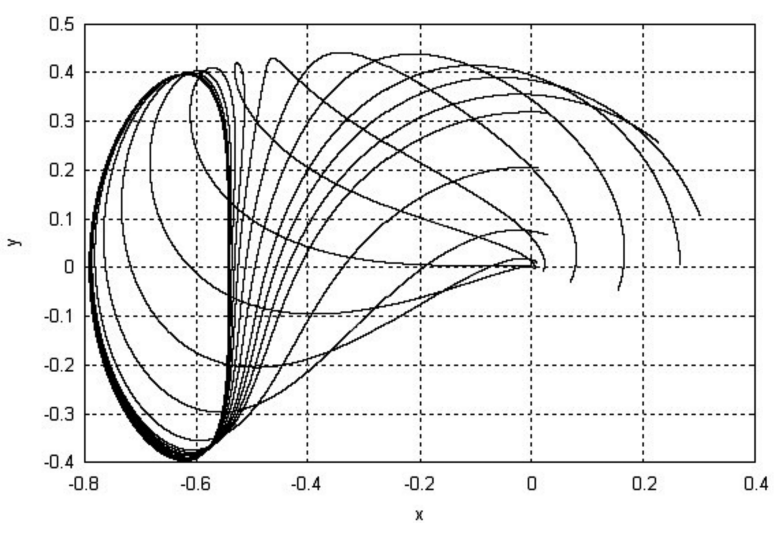

Fig. 3 Stable manifold around $L 1$ (until first periapsis point). space and have a close flyby to the target body. At closest approach, an impulsive maneuver is performed to put the spacecraft on the stable manifold that leads to capture to a periodic orbit of $L 1$ or $L 2$ of the target body. The reason why an impulsive maneuver is performed near the surface of the target body (periapsis) is because this is the energetically most efficient place to reduce the approach energy, which may also be reduced by using an aeroassist with the planetary atmosphere. Once placed on the stable manifold, the S/C approaches the periodic orbit, perhaps orbiting around the target body a few times on its way. Once it is close to and crosses the periodic orbit, an infinitesimal impulsive maneuver is necessary to place it on the periodic orbit. The cost of this final maneuver is negligible and is ignored. In this way, the stable manifolds are used for capture trajectories to periodic orbits.

\section{B. Definition of Periapsis Points}

In this section, we investigate the first four periapsis passage points of stable manifolds, where an impulse maneuver may be performed, propagated backward from a certain point on the periodic orbits in time. Figure 4 shows an example of the first four periapsis passage points of one example trajectory of the $L 1$ stable manifold (in the planar case for $J=-2.15)$. The secondary body is located at the origin. Based on this result, Figs. 5 and 6 plot the first four periapsis points' locations of the $L 1$ stable manifold for several values of the Jacobi constant (i.e., the size of periodic orbit) in the twodimensional (Lyapunov orbits) and three-dimensional (Halo orbits) cases, respectively. The periapsis locations of $L 1$ and $L 2$ stable manifolds are symmetric to the $x=0$ plane. We can see that each periapsis point region spreads out, and that these periapsis locations depend on the value of the Jacobi constant.

Figures 7 and 8 show the relation between a minimum periapsis distance and the value of the Jacobi constant in the two- and threedimensional cases, respectively. The minimum periapsis distance means the distance from the origin to the periapsis point of stable manifold, which is closest to the origin in each of four periapsis points in the same value of the Jacobi constant in the twodimensional cases. The minimum periapsis distance decreases as the value of the Jacobi constant increases, and each of the four minimum periapsis distances become smaller than 0.000148 (which is smaller than the smallest normalized planetary radius, Neptune) when the value of the Jacobi constant is large. Thus, the stable manifold of the

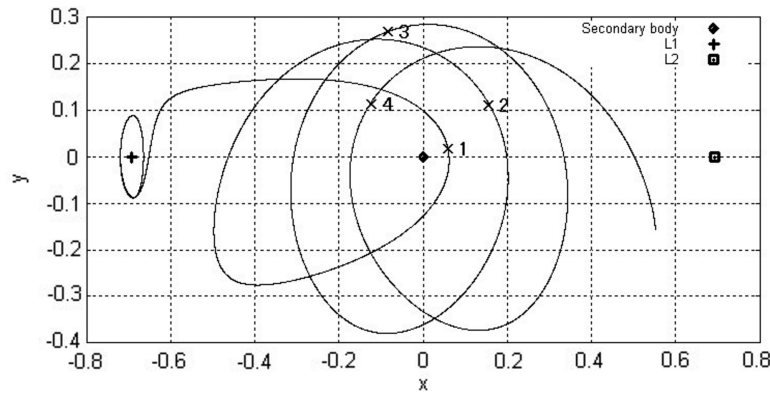

Fig. 4 First four periapsis points of an example trajectory of the $L 1$ stable manifold propagated backward from a certain point on the periodic orbits. 


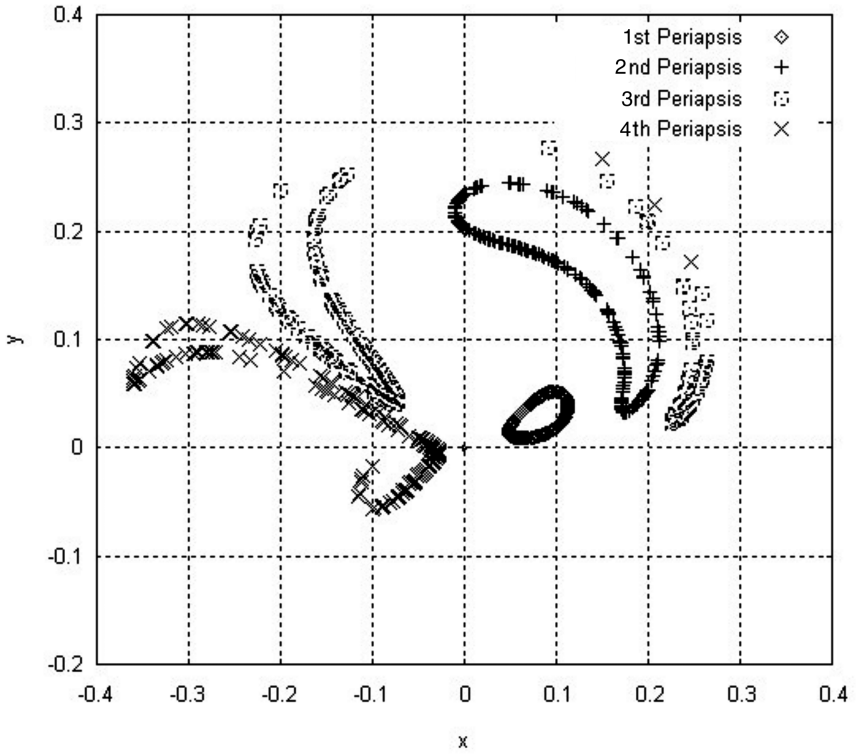

a) $\mathrm{J}=-\mathbf{2 . 1 5}$

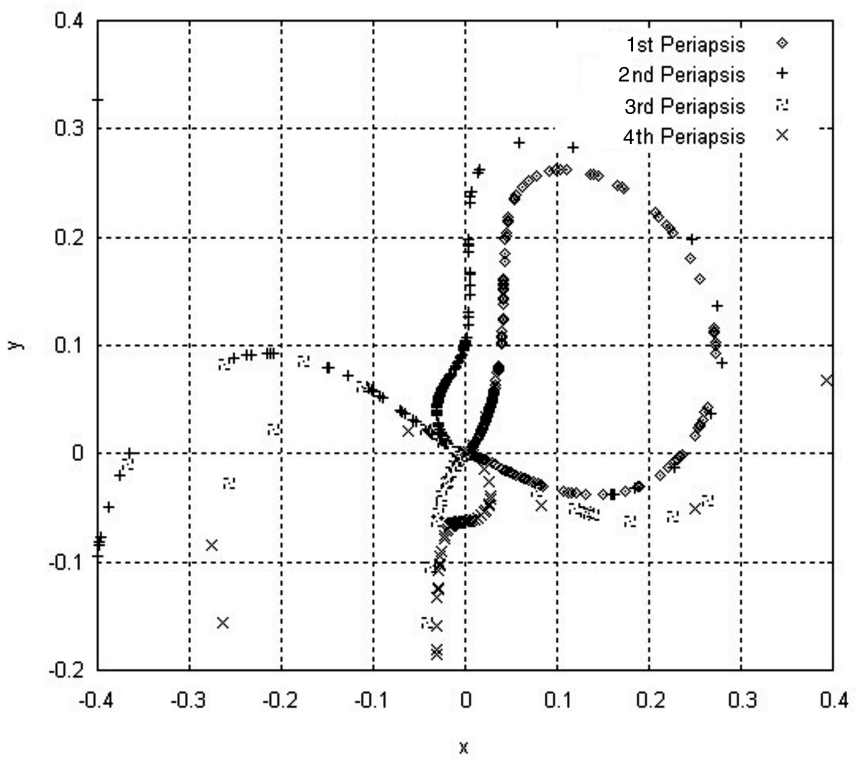

c) $\mathbf{J}=\mathbf{- 1 . 9 5}$

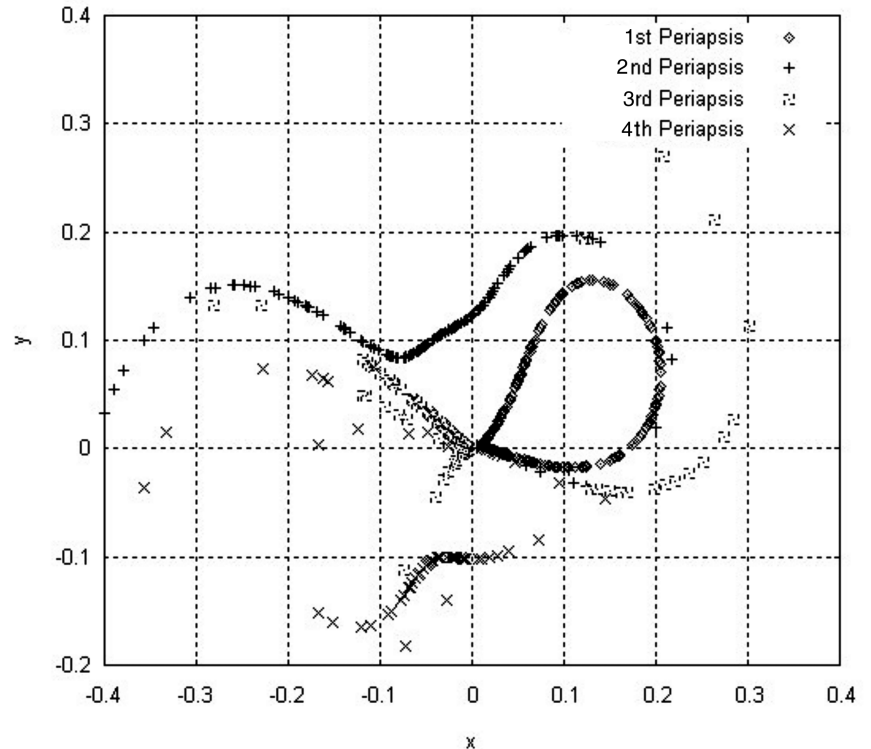

b) $\mathbf{J}=\mathbf{- 2 . 0 5}$

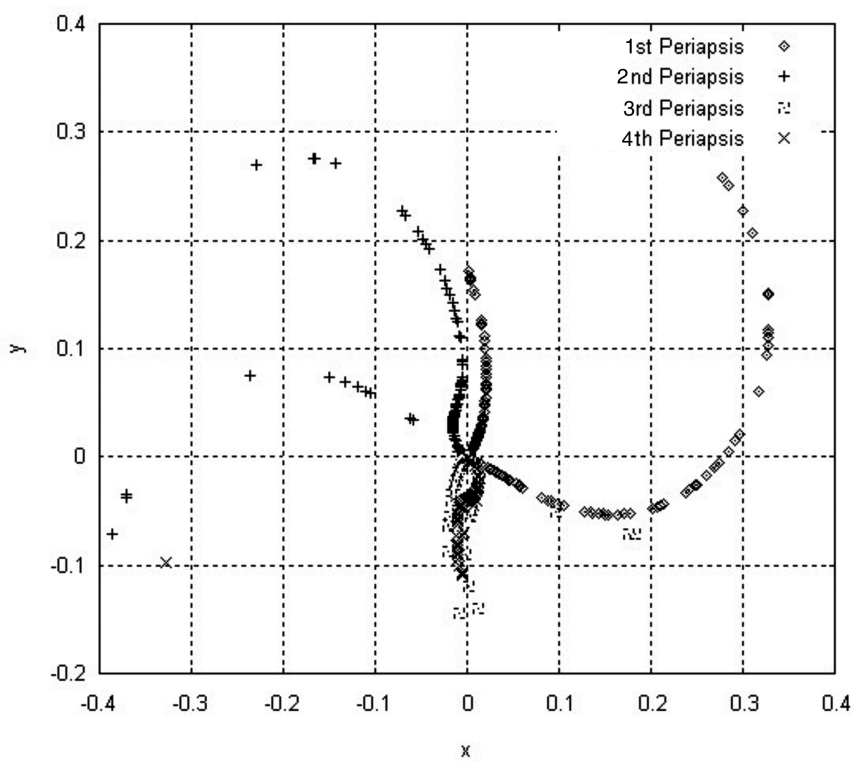

d) $\mathbf{J}=\mathbf{- 1 . 8 5}$

Fig. 5 First four periapsis locations of $L 1$ stable manifold in the two-dimensional case; the secondary body is located at the origin.

first four periapsis passage points can intersect the surface of any of the planets in the solar system. That is to say, a spacecraft can be placed onto the stable manifold of a Lyapunov orbit by performing an impulsive maneuver at the aforementioned periapsis points. In the three-dimensional case, the minimum periapsis distance depends on the value of the Jacobi constant as well, and although the value of the Jacobi constant is limited, those distances become smaller than 0.000148 . Therefore, the stable manifold of the first four periapsis passage points can intersect the surface of any of the planets in the solar system in the three-dimensional case.

\section{Application to Earth-Mars Transfer}

In this section, a patched conic approximation for the interplanetary transfer is applied to our study of capture trajectories to periodic orbits around libration points in both two- and threedimensional cases. We focus our attention on a transfer from Earth to Mars. However, these results can be applied to other planets of the solar system as well. First, we compare the total $\Delta V$ after an interplanetary transfer between the following cases: one is a direct entry into a target circular orbit of Mars at a low altitude
( $h \cong 200 \mathrm{~km}$ ), and the other is an entry to the target circular orbit after first inserting into a periodic orbit around libration points. We also compare the cost of transfer into the Libration orbit to the cost of capture to Mars, assuming the two-body problem only.

\section{A. Two-Dimensional Case}

If we assume a simple Hohmann transfer from Earth to Mars, the hyperbolic arrival velocity with respect to Mars is $v_{\infty, a}=2.648 \mathrm{~km} / \mathrm{s}$. Subsequently, the velocity in the hyperbolic trajectory at periapsis (altitude $h \cong 200 \mathrm{~km}$ ) to Mars is $v_{p / M}=$ $\sqrt{v_{\infty, a}^{2}+2 \mu_{M} /\left(r_{M}+h\right)}=5.551 \mathrm{~km} / \mathrm{s}\left(\mu_{M}\right.$ is the gravitational parameter of Mars and $r_{M}$ is the radius of Mars). The velocity in a circular orbit at the altitude $h \cong 200 \mathrm{~km}$ of Mars is $v_{c / M}=\sqrt{\mu_{M} /\left(r_{M}+h\right)}=3.449 \mathrm{~km} / \mathrm{s}$. Hence, in the direct entry case, the required $\Delta V_{c / M}$ for entry in the circular orbit (i.e., total $\Delta V$ ) is $5.551-3.449=2.102 \mathrm{~km} / \mathrm{s}$.

Next, we consider an entry into a circular orbit of Mars via Lyapunov orbit case. When the value of the Jacobi constant $J_{M}$ is -1.898 , for example, the third periapsis point of the stable manifold associated with the $L 1$ Lyapunov orbit pass at a $200 \mathrm{~km}$ altitude with 


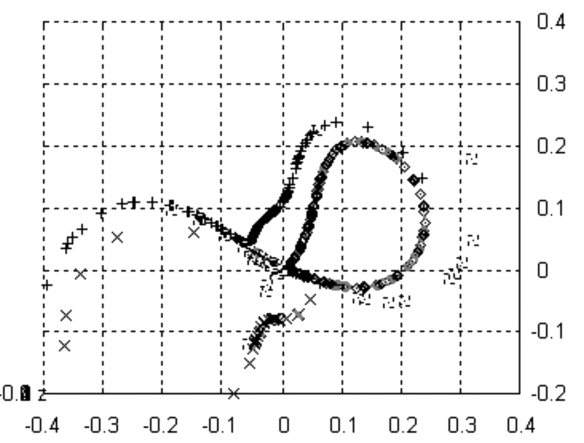

y

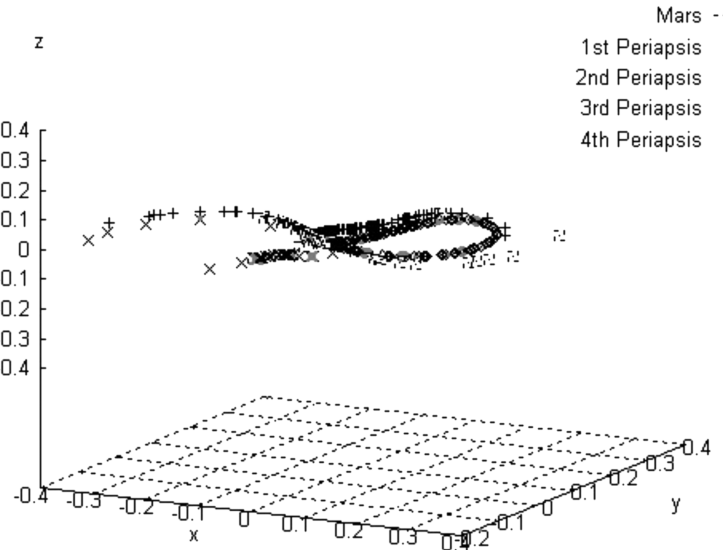

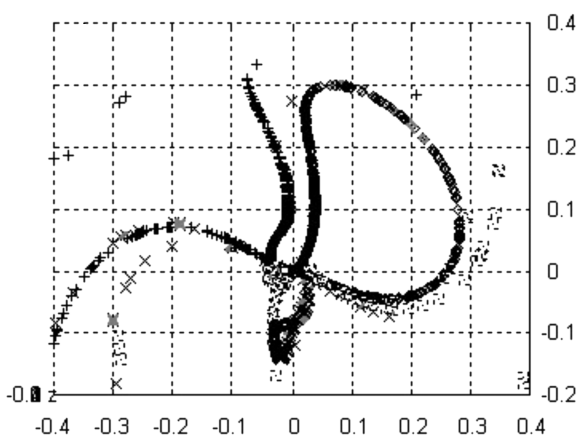
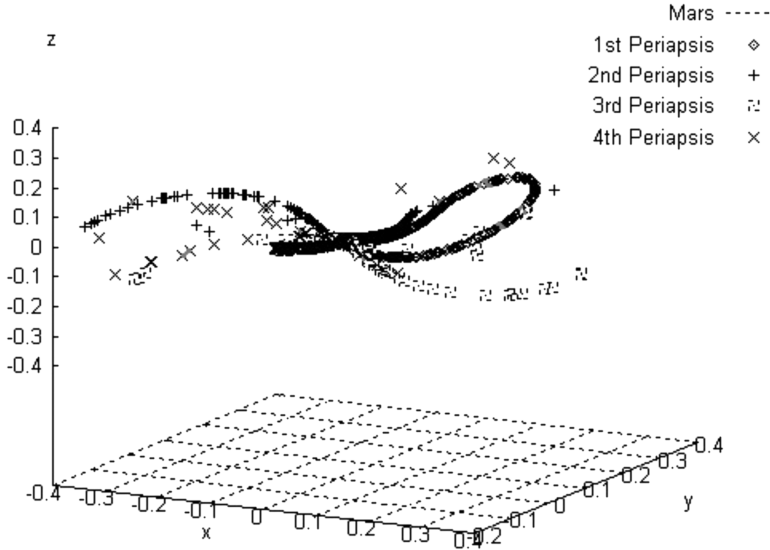

b) $\mathbf{J}=\mathbf{- 1 . 8 4}$

a) $\mathbf{J}=\mathbf{- 2 . 0 1}$
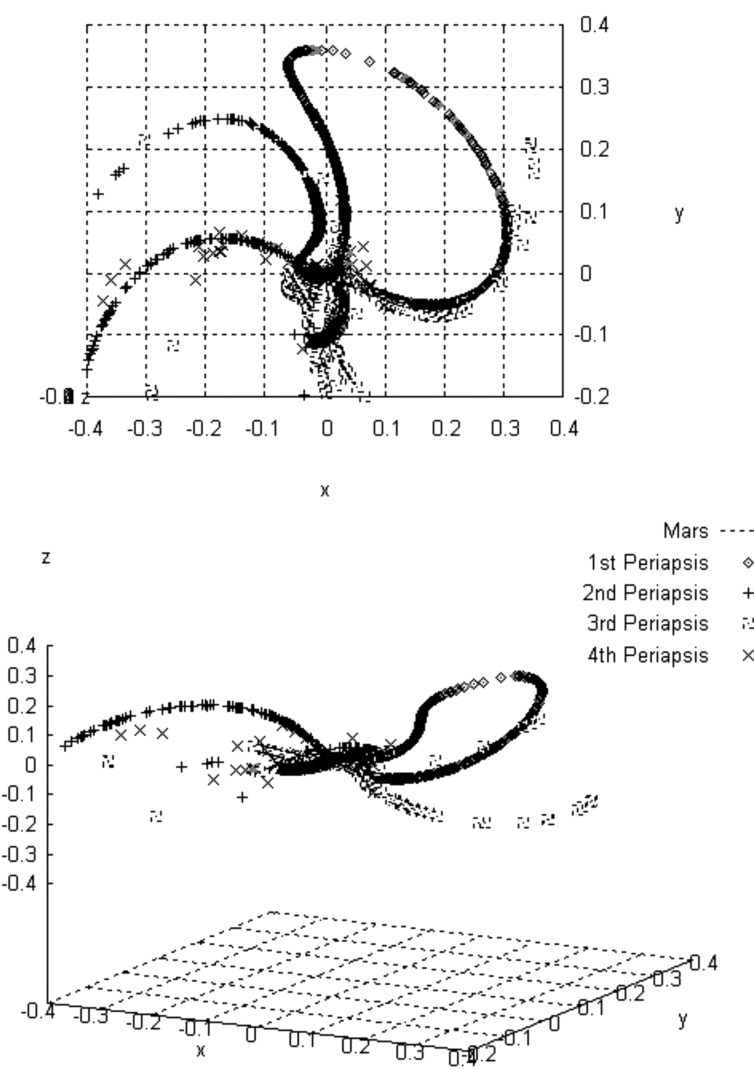

c) $\mathbf{J}=-\mathbf{- 1 . 7 5}$

Fig. 6 First four periapsis locations of $L 1$ stable manifold in the three-dimensional case; the secondary body is located at the origin.

respect to Mars. The velocity in the $L 1$ stable manifold at the third periapsis point in the areocentric frame is $5.009 \mathrm{~km} / \mathrm{s}$. Therefore, the minimum required $\Delta V_{p / M}$ for entry to the $L 1$ Lyapunov orbit with $J_{M}=-1.898$ is $5.551-5.009=0.542 \mathrm{~km} / \mathrm{s}$. Here, it is instructive to compare the $\Delta V$ for capture to the periodic orbit with simple two- body capture criterion at Mars: $\Delta V_{p / M}=v_{p / M}-2^{1 / 2} v_{c / M}=$ $0.673 \mathrm{~km} / \mathrm{s}$. Thus, capture to the periodic orbit allows a significant improvement even with respect to capture into a parabolic orbit, or, in other words, after our capture maneuver is performed, the spacecraft is initially on a hyperbolic orbit, but one that transitions to a bound 


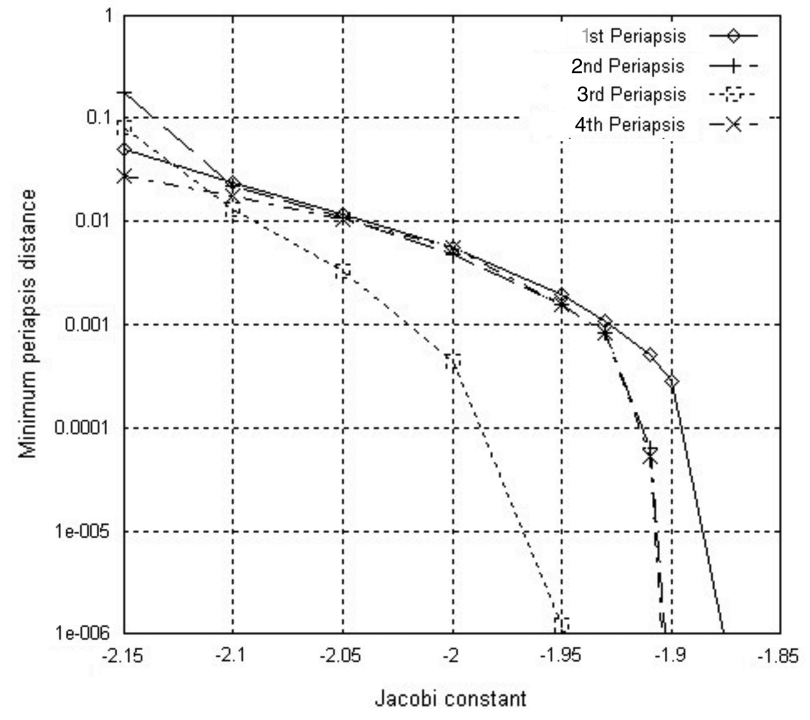

Fig. 7 Minimum periapsis distance as a function of the value of Jacobi constant (the size of Lyapunov orbit) in the two-dimensional case.

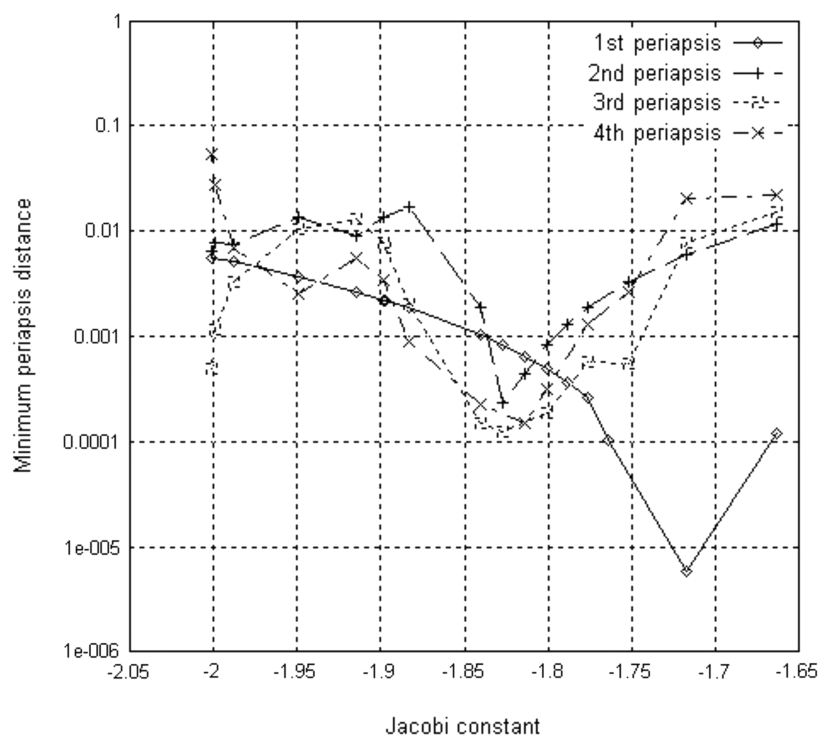

Fig. 8 Minimum periapsis distance as a function of the value of Jacobi constant (the size of Halo orbit) in the three-dimensional case.

periodic orbit. After entering the L1 Lyapunov orbit, the S/C can leave from that orbit with virtually no cost and approach Mars on the unstable manifold to that orbit. If the departure time is chosen correctly, the velocity on the $L 1$ unstable manifold at a periapsis point of Mars is $5.009 \mathrm{~km} / \mathrm{s}$. Thus, the minimum required $\Delta V_{c / M}$ to enter into the $200 \mathrm{~km}$ circular orbit of Mars is $5.009-3.449=1.560 \mathrm{~km} / \mathrm{s}$. Therefore, the total $\Delta V$ via the $L 1$ Lyapunov orbit case is $0.542+1.560=2.102 \mathrm{~km} / \mathrm{s}$ and is identical to the value of the direct entry case (see Table 2), as is expected. However, if a spaceport is built on the Lyapunov orbit, fuel

Table 2 Required $\Delta V$ to capture to orbit around Mars

\begin{tabular}{lccc}
\hline \hline & $\begin{array}{r}\Delta V_{p / M}, \\
\mathrm{~km} / \mathrm{s}\end{array}$ & $\begin{array}{c}\Delta V_{c / M}, \\
\mathrm{~km} / \mathrm{s}\end{array}$ & $\begin{array}{c}\text { total } \Delta V, \\
\mathrm{~km} / \mathrm{s}\end{array}$ \\
\hline Direct entry & - & 2.102 & 2.102 \\
Entry via $L$ 1 Lyapunov orbit & 0.542 & 1.560 & 2.102 \\
$\begin{array}{l}\text { Entry via } L \text { 2 Halo orbit } \\
\text { (Two-body capture to } \\
\text { parabolic orbit) }\end{array}$ & 0.539 & 1.563 & 2.102 \\
\hline \hline
\end{tabular}

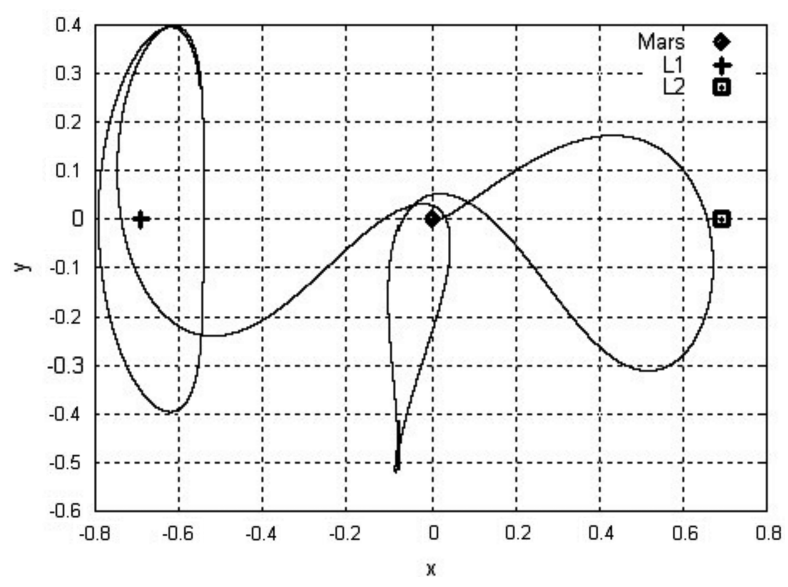

Fig. 9 Capture trajectory from the periapsis point of Mars to the $L 1$ Lyapunov orbit.

can be supplied there or payloads are transferred from interplanetary cargo ship to a space vehicle exclusively used at Mars so that $\Delta V_{c / M}$ is reduced. Figure 9 shows this capture trajectory from the third periapsis point of Mars to the Lyapunov orbit.

\section{B. Three-Dimensional case}

Now, we examine the three-dimensional case. In analogy with the two-dimensional case, assuming $v_{\infty, a}=2.648 \mathrm{~km} / \mathrm{s}$, the velocity in the hyperbolic trajectory at periapsis (altitude $h \cong 200 \mathrm{~km}$ ) to Mars $v_{p / M}$ can again be estimated at $5.551 \mathrm{~km} / \mathrm{s}$, and the velocity in a $200 \mathrm{~km}$ circular orbit can again be $V_{c / M}=3.449 \mathrm{~km} / \mathrm{s}$. Thus, in the direct entry case, the required $\Delta V_{c / M}$ for entry into the circular orbit (i.e., total $\Delta V$ ) is $5.676-3.449=2.102 \mathrm{~km} / \mathrm{s}$.

Next, in the via Halo orbit case, the velocity at the second periapsis passage point (altitude $h \cong 200 \mathrm{~km}$ of Mars) of the $L 2$ stable manifold associated with the Halo orbit $(J=-1.752)$ is $5.012 \mathrm{~km} / \mathrm{s}$ in the areocentric frame. Therefore, the minimum required $\Delta V_{p / M}$ for entry to the $L 2$ Halo orbit of $J_{\mathrm{HO}}=-1.752$ is approximately $5.551-5.012=0.539 \mathrm{~km} / \mathrm{s}$. Like the two-dimensional case, the caption to the periodic orbit is improved compared with the two-body capture into a parabolic orbit. Moreover, the minimum required $\Delta V_{c / M}$ to enter into the circular orbit around Mars leaving from the Halo orbit without a station keeping control is $5.012-3.449=1.563 \mathrm{~km} / \mathrm{s}$. Finally, the total $\Delta V$ via the $L 2$ Halo orbit case is $0.539+1.563=2.102 \mathrm{~km} / \mathrm{s}$. These results are summarized in Table 2. As before, if a spaceport is built on the Halo orbit so that the fuel can be supplied there, or payloads are a transferred from interplanetary cargo ship to a space vehicle exclusively used at Mars, the required $\Delta V_{c / M}$ needed to be carried by the interplanetary vehicle for entry into a circular orbit from an interplanetary trajectory can again be reduced compared with the direct entry case. Figure 10 shows these capture trajectories from the second periapsis point of Mars to the $L 2$ Halo orbits.

\section{Conclusions}

This paper investigates capture trajectories to Lyapunov/ Halo orbits in the Hill three-body problem. We concentrate on transfers into these vicinities from interplanetary trajectories. The characteristics of the relation between the minimum periapsis distance and the size of Lyapunov/Halo orbits are obtained using the manifold trajectories from Lyapunov/Halo orbits by backward integration. Numerical results show that the manifold trajectories of the periapsis passage points from Lyapunov/Halo orbits can intersect the surface of any of the planets in the solar system if the value of the Jacobi integral is changed. Thus, this technique can be used to reduce the cost of capture at any of the planets in the solar system.

As an example, we consider transfers from an interplanetary transfer into a target circular orbit near the surface of Mars. We discussed three cases: a direct transfer into the target circular orbit 


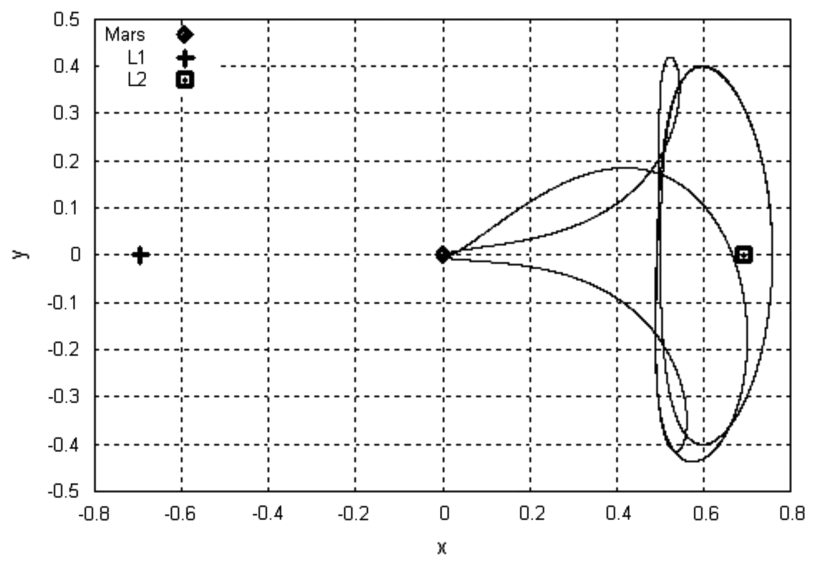

a) $x-y$ plane

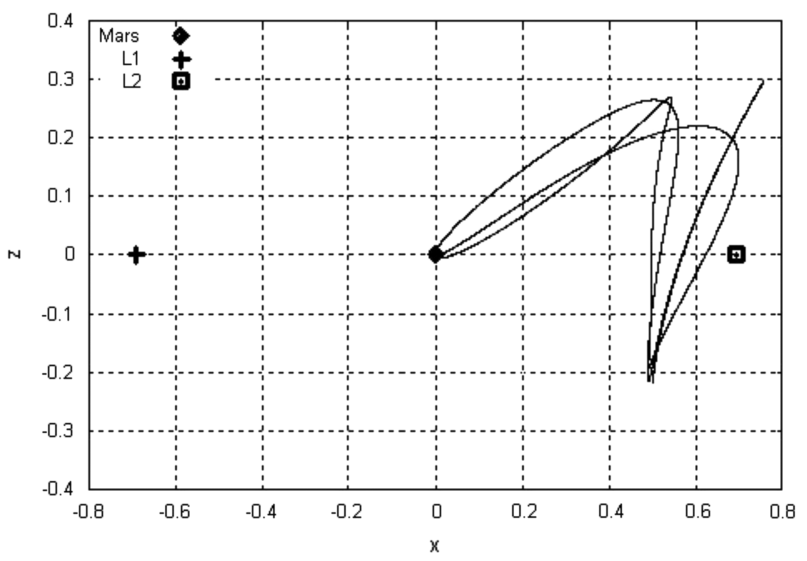

b) $x-z$ plane

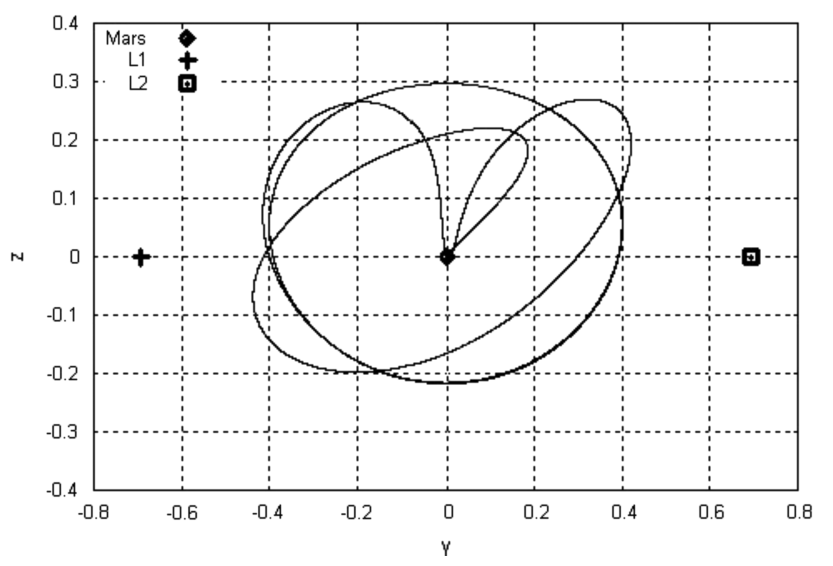

c) y-z plane

Fig. 10 Capture trajectory from the periapsis point of Mars to the $L 2$ Halo orbit.

around Mars, a transfer to the target circular orbit after going into a Lyapunov/Halo orbit by performing a velocity correction near the surface of Mars, and direct capture into a parabolic orbit about Mars. It was found that if a spaceport is built on the Lyapunov/Halo orbit and the fuel could be supplied there, the required velocity increment for entry into the circular orbit from the interplanetary trajectory could be reduced compared with the direct entry case.

\section{References}

[1] D'Amario, L. A., and Edelbaum, T. N., "Minimum Impulse ThreeBody Trajectories," AIAA Journal, Vol. 12, No. 4, 1974, pp. 455-462.

[2] Broucke, R., "Traveling Between the Lagrange Points and the Moon," Journal of Guidance and Control, Vol. 2, No. 4, 1979, pp. 257-263.

[3] Gomez, G., Jorba, A., Masdemont, J., and Simo, C., "Study of the Transfer from the Earth to a Halo Orbit Around the Equilibrium Point L1," Celestial Mechanics and Dynamical Astronomy, Vol. 56, No. 4,
1993, pp. $541-562$ doi:10.1007/BF00696185

[4] Prado, A. F. B. A., "Travelling Between the Lagrange Points and the Earth," Acta Astronautica, Vol. 39, No. 7, 1996, pp. 483-486. doi:10.1016/S0094-5765(97)85428-8

[5] Strizzi, J. D., Kutrieb, J., Damphousse, P., and Carrico, J., "Sun-Mars Libration Points and Mars Mission Simulations," AAS/AIAA Spaceflight Mechanics Meeting, American Astronautical Society Paper 01159, Feb. 2001.

[6] Baoyin, H., and McInnes, R. C., "Trajectories to and from the Lagrange Points and the Primary Body Surfaces," Journal of Guidance, Control, and Dynamics, Vol. 29, No. 4, 2006, pp. 998-1003. doi: $10.2514 / 1.17757$

[7] Canalias, E., Gomez, G., Marcote, M., and Masdemont, J. J., "Assessment of Mission Design Including Utilization of Libration Points and Weak Stability Boundaries," ESA Advanced Concepts Team, http://www.esa.int/act [cited 20 May 2008].

[8] Folta, D. C., Beckman, M., Leete, S. J., Marr, G. C., Mesarch, M., and Cooley, S., "Servicing and Deployment of National Resources in SunEarth. Libration-Point Orbits," International Astronautical Congress Paper 02-Q.6.08, Oct. 2002.

[9] Lo, M., and Ross, S., "Lunar $L 1$ Gateway: Portal to the Stars and Beyond," AIAA Paper 2001-4768, Aug. 2001.

[10] Marsden, J. E., and Ross, S. D., "New Methods in Celestial Mechanics and Mission Design," Bulletin of the American Mathematical Society, Vol. 43, No. 1, 2006, pp. 43-73. doi:10.1090/S0273-0979-05-01085-2

[11] Farquhar, R. W., "Future Missions for Libration-Point Satellites," Astronautics and Aeronautics, Vol. 7, No. 5, May 1969, pp. 52-56.

[12] Farquhar, R. W., Dunham, D. W., Guo, Y., and Madams, V. J., "Utilization of Libration Points for Human Exploration in the SunEarth-Moon System and Beyond," Acta Astronautica, Vol. 55, Nos. 39, 2004, pp. 687-700. doi:10.1016/j.actaastro.2004.05.021

[13] Conley, C. C., "Low Energy Transit in the Restricted Three Body Problem," SIAM Journal on Applied Mathematics, Vol. 16, No. 4, 1968, pp. 732-746. doi: $10.1137 / 0116060$

[14] Koon, W. S., Lo, M. W., Marsden, J. E., and Ross, S. D., "Heteroclinic Connections Between Periodic Orbits and Resonance Transitions in Celestial Mechanics," Chaos, Vol. 10, No. 2, 2000, pp. 427-469. doi: $10.1063 / 1.166509$

[15] Koon, W. S., Lo, M. W., Marsden, J. E., and Ross, S. D., "Constructing a Low Energy Transfer Between Jovian Moons Contemporary," Contemporary Mathematics (American Mathematical Society), Vol. 292, No. 1, 2002, pp. 129-145.

[16] Lo, M., "Interplanetary Superhighway and the Origins Program," IEEE Space 2002 Conference, Inst. of Electrical and Electronics Engineers, Piscataway, NJ, March 2002.

[17] Yamato, H., and Spencer, D. B., "Transit-Orbit Search for Planar Restricted Three-Body Problems with Perturbations.," Journal of Guidance, Control, and Dynamics, Vol. 27, No. 6, 2004, pp. 1035 1045 . doi: $10.2514 / 1.4524$

[18] Topputo, F., Vasile, M., and Bernelli-Zazzera, F., "Low Energy Interplanetary Transfers Exploiting Invariant Manifolds of the Restricted Three Body Problem," Journal of the Astronautical Sciences, Vol. 53, No. 4, 2005, pp. 353-372.

[19] Howell, K., and Kakoi, M., "Transfers Between the Earth-Moon and Sun-Earth Systems Using Manifolds and Transit Orbits," Acta Astronautica, Vol. 59, Nos. 1-5, 2006, pp. 367-380. doi:10.1016/j.actaastro.2006.02.010

[20] Matsumoto, M., and Kawaguchi, J., "Deep Space Quay and Solar Voyage Era," Sixth IAA International Conference on Low-Cost Planetary Missions, International Academy of Astronautics, 2005, pp. 401-408.

[21] Nakamiya, M., and Yamakawa, H., "Analysis of Earth Escape Trajectories Starting from Sun-Earth $L 2$ Point," Proceedings of the 16th Workshop on Astrodynamics and Flight Mechanics, Inst. of Space and Astronautical Science (Japan), 2006, pp. 42-48.

[22] Nakamiya, M., and Yamakawa, H., "Earth Escape Trajectories Starting from L2 Point," AIAA/AAS Astrodynamics Specialist Conference and Exhibit, AIAA Paper 2006-6751, 2006.

[23] Belbruno, E., and Miller, J., "Sun-Perturbed Earth-to-Moon Transfers with Ballistic Capture," Journal of Guidance, Control, and Dynamics, Vol. 16, No. 4, 1993, pp. 770-775.

[24] Yamakawa, H., Kawaguchi, J., Ishii, N., and Matsuo, H., "On EarthMoon Transfer Trajectory with Gravitational Capture," American Astronautical Society, Paper AAS 93-633, Aug. 1993. 
[25] Brunini, A., "On the Satellite Capture Problem Capture and Stability Regions for Planetary Satellites," Celestial Mechanics and Dynamical Astronomy, Vol. 64, Nos. 1-2, 1996, pp. 79-92. doi:10.1007/BF00051607

[26] Howell, K. C., Marchand, B. G., and Lo, M. W., "Temporary Satellite Capture of Short-Period Jupiter Family Comets from the Perspective of Dynamical Systems," Journal of the Astronautical Sciences, Vol. 49, No. 4, 2001, pp. 539-557.

[27] Makó, Z., and Szenkovits, F., "Capture in the Circular and Elliptic Restricted Three-Body Problem," Celestial Mechanics and Dynamical Astronomy, Vol. 90, Nos. 1-2, 2004, pp. 51-58. doi:10.1007/s10569-004-5899-7

[28] Paskowitz, M. E., and Scheeres, D. J., "Robust Capture and Transfer Trajectories for Planetary Satellite Orbiters," Journal of Guidance, Control, and Dynamics, Vol. 29, No. 2, 2006, pp. 342-353. doi:10.2514/1.13761

[29] Scheeres, D., Guman, M., and Villac, B., "Stability Analysis of Planetary Satellite Orbiters: Application to the Europa Orbiter," Journal of Guidance, Control, and Dynamics, Vol. 24, No. 4, 2001, pp. 778-787.

[30] Szebehely, V., Theory of Orbits: The Restricted Problem of Three Bodies, Academic Press, New York, 1967.

[31] Villac, B. F., and Scheeres, D. J., "Escaping Trajectories in the Hill Three-Body Problem and Applications," Journal of Guidance, Control, and Dynamics, Vol. 26, No. 2, 2003, pp. 224-232.

[32] Henon, M., "Numerical Exploration of the Restricted Problem, V. Hill's Case: Periodic Orbits and Their Stability," Astronomy and
Astrophysics Vol. 1, No. 2, 1969, pp. 223-238.

[33] Breakwell, J. V., and Brown, J. V., "'Halo' Family of 3-Dimensional Periodic Orbits in the Earth-Moon Restricted 3-Body Problem," Celestial Mechanics, Vol. 20, No. 4, Nov. 1979, pp. 389404.

doi:10.1007/BF01230405

[34] Farquhar, R. W., "Control and Use of Libration-Point Satellites," NASA TR R-346, 1970.

[35] Richardson, D. L., "Analytic Construction of Periodic Orbits About the Collinear Points," Celestial Mechanics, Vol. 22, Oct. 1980, pp. 241253. doi:10.1007/BF01229511

[36] Howell, K. C., "Families of Orbits in the Vicinity of the Collinear Libration Points," Journal of the Astronautical Sciences, Vol. 49, No. 1, Jan.-March 2001, pp. 107-125.

[37] Lawrence, C., Zhou, J., and Tits, A., "User's Guide for CFSQP Ver. 2.5: A C Code for Solving (Large Scale) Constrained Nonlinear (Minimax) Optimization Problems, Generating Iterates Satisfying All Inequality Constraints," Inst. for Systems Research, Univ. of Maryland, TR 94 16r1, College Park, MD, 1997.

[38] Simo, C., and Stuchi, T., "Central Stable/Unstable Manifolds and the Destruction of KAM Tori in the Planar Hill Problem," Physica D: Nonlinear Phenomena, Vol. 140, Nos. 1-2, 2000, pp. 1-32. doi:10.1016/S0167-2789(99)00211-0

[39] Gomez, G., Marcote, M., and Mondelo, J., "Invariant Manifold Structure of the Spatial Hill's Problem," Dynamical Systems: An International Journal, Vol. 20, No. 1, 2005, pp. 115-147. 